\title{
Effect of Oral Hydration on Contrast-Induced Acute Kidney Injury among Patients after Primary Percutaneous Coronary Intervention
}

\author{
Weining Xie ${ }^{a}$ Yuge Zhou $^{b}$ Zhishan Liao ${ }^{c}$ Biying Lin ${ }^{d}$ \\ aDepartment of Scientific Research, Guangdong Province Hospital of Integrated Traditional Chinese and Western \\ Medicine, Foshan, China; ${ }^{b}$ Affiliated Guangdong Hospital of Integrated Traditional Chinese and Western Medicine \\ of Guangzhou University of Chinese Medicine, Foshan, China; 'Department of Cardiology, Guangdong Province \\ Hospital of Integrated Traditional Chinese and Western Medicine, Foshan, China; 'Department of Nephrology, \\ Guangdong Province Hospital of Integrated Traditional Chinese and Western Medicine, Foshan, China
}

\section{Keywords}

Oral hydration - ST-elevation myocardial infarction .

Contrast-induced acute kidney injury

\begin{abstract}
Objectives: The purpose of this study was to evaluate the protective effect of oral hydration volume to weight ratio (OHV/W) on contrast-induced acute kidney injury (Cl-AKI) among patients with ST-elevation myocardial infarction (STEMI) following percutaneous coronary intervention ( $\mathrm{PCI}$ ). Methods: A total of 754 patients with STEMI undergoing $\mathrm{PCl}$ were selected. Each patient was encouraged to drink as much water as possible $24 \mathrm{~h}$ after $\mathrm{PCl}$. Total volume intake was recorded for all patients. The ratio of $\mathrm{OHV} / \mathrm{W}$ was calculated. The occurrence of $\mathrm{Cl}-\mathrm{AKI}$ was defined as $\geq 0.5 \mathrm{mg} / \mathrm{dL}$ absolute or $\geq 25 \%$ relative increase in serum creatinine within 48-72 $\mathrm{h}$ following PCl. Logistic regression analysis and generalized additive model were performed to evaluate the relationship between $\mathrm{OHV} / \mathrm{W}$ and $\mathrm{Cl}-\mathrm{AKI}$. Results: There was a nonlinear relationship between $\mathrm{OHV} / \mathrm{W}$ and $\mathrm{Cl}-\mathrm{AKI}$ with an inflection point of $15.69 \mathrm{~mL} / \mathrm{kg}$. On the right side of the inflection point $(\mathrm{OHV} / \mathrm{W} \geq 15.69 \mathrm{~mL} / \mathrm{kg}$ ), a negative relationship was detected between $\mathrm{OHV} / \mathrm{W}$ and $\mathrm{Cl}-\mathrm{AKI}(\mathrm{HR}=0.90$, $95 \% \mathrm{Cl}: 0.82 \sim 0.98, p=0.0126)$. However, no relationship was
\end{abstract}

karger@karger.com www.karger.com/crm

Karger $\stackrel{\text { ' }}{5}$
(C) 2021 The Author(s)

Published by S. Karger AG, Basel

This is an Open Access article licensed under the Creative Commons Attribution-NonCommercial-4.0 International License (CC BY-NC) (http://www.karger.com/Services/OpenAccessLicense), applicable to the online version of the article only. Usage and distribution for commercial purposes requires written permission. observed between $\mathrm{OHV} / \mathrm{W}$ and $\mathrm{Cl}-\mathrm{AKI}$ on the left of inflection point $(H R=1.19,95 \% \mathrm{Cl}: 0.95 \sim 1.49, p=0.1302)$. Subgroup analysis showed that significant interactions were observed only for gender difference ( $p$ for interaction $=0.0155$ ), male patients had a significantly lower risk of $\mathrm{Cl}-\mathrm{AKI}(\mathrm{HR}=0.84$, 95\% Cl: 0.75 0.93, $p=0.0012$ ). Conclusion: $\mathrm{OHV} / \mathrm{W} \geq 15.6$ $\mathrm{mL} / \mathrm{kg}$ for $24 \mathrm{~h}$ post-procedure may be an effective preventive strategy of $\mathrm{Cl}-\mathrm{AKI}$. In addition, male patients may particularly benefit from $\mathrm{OHV}$ to prevent $\mathrm{Cl}-\mathrm{AKI}$.

(C) 2021 The Author(s) Published by S. Karger AG, Basel

\section{Introduction}

Contrast-induced acute kidney injury (CI-AKI) is one of the common causes of hospital-acquired acute renal insufficiency, which is caused by intravascular injection of contrast media (CM) $[1,2]$. The risk of CI-AKI is associated with the increase of CM volume [3] and contributes toward poorer clinical outcomes $[4,5]$. At the same time, with the increasing frequency of primary percutaneous coronary intervention (PCI), the incidence of CIAKI gradually increases, ranging from $2 \%$ of patients with adequate renal function at baseline to $20-30 \%$ of pa- 
Fig. 1. Flowchart of patient selection. CIAKI, contrast-induced acute kidney injury; PCI, percutaneous coronary intervention.

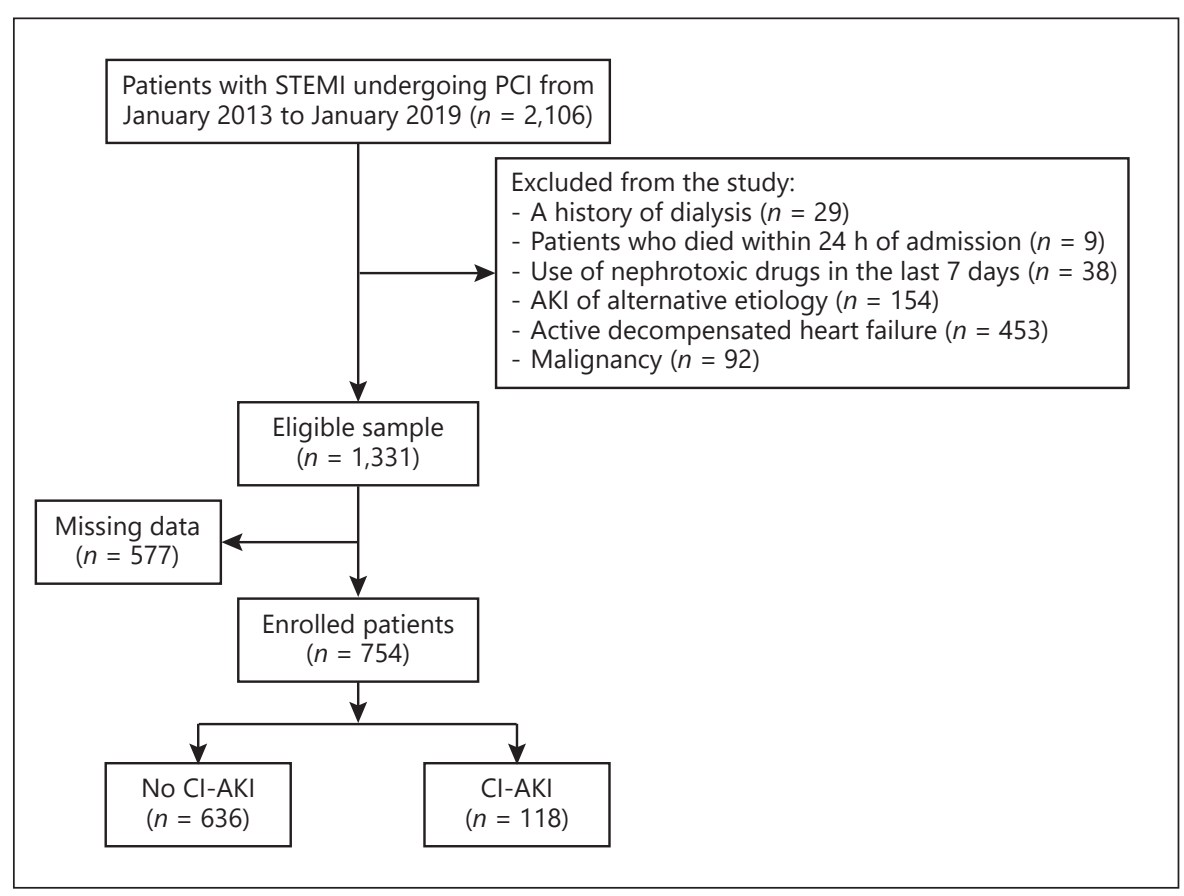

tients with chronic kidney disease [6]. Today, the strategies to prevent CI-AKI include fluid expansion, application of a hypotonic or isotonic contrast agents, and minimization of $\mathrm{CM}$ dose. Fluid expansion includes intravascular volume expansion and oral volume expansion [7-10], and intravascular volume expansion has been accepted as the cornerstone to prevent CI-AKI [10]. However, there is limited information on oral volume expansion for the prevention of CI-AKI risk in patients with ST-elevation myocardial infarction (STEMI). Compared with selective PCI [11], patients with STEMI treated by primary PCI have a higher risk of CI-AKI due to the use of large amounts of contrast agent, and patients with STEMI have an urgent need for surgery and lack of effective preventive measures [12-14]. In the present study, we aimed to explore the value of post-procedural oral hydration in preventing CI-AKI risk in STEMI patients flowing PCI.

\section{Methods}

\section{Research Subjects}

This was a single-center, retrospective, observational study. A total of 754 consecutive patients aged $\geq 18$ years with STEMI undergoing PCI from January 2013 to January 2019 were selected from Guangdong Province Hospital of Integrated Traditional Chinese and Western Medicine. The diagnosis of STEMI was in accordance with STEMI diagnostic criteria [15]: (1) Sustained chest pain $>30 \mathrm{~min}$; (2) ST-segment elevation in $\geq 2$ contiguous leads or new left bundle-branch block; (3) elevated cardiac troponin $\mathrm{T}$ within $24 \mathrm{~h}$ of symptoms onset. Exclusion criteria included: (1) a history of dialysis; (2) patients who died within $24 \mathrm{~h}$ of admission; (3) use of nephrotoxic drugs in the last 7 days; (4) AKI of alternative etiology; (5) active decompensated heart failure; (6) malignancy; (7) missing data (Fig. 1). The study was approved by the Ethics Committee of Guangdong Province Hospital of Integrated Traditional Chinese and Western Medicine. This was a retrospective study, so informed consent from patients was not required.

\section{Measurement}

The blood pressure was measured after at least 15 min of rest. Body weight and standing height were measured. Body mass index (BMI) (calculated as weight in kilograms divided by height in meters squared). Venous blood samples were collected on the day of admission and every $48-72 \mathrm{~h}$ after the procedure and at hospital discharge. Serum creatinine (Scr), blood urea nitrogen, total cholesterol, triglyceride, low-density lipoprotein cholesterol, highdensity lipoprotein cholesterol, and uric acid levels were measured using Olympus AU-640 autoanalyzer (Olympus, Shinjuku-ku, Japan). Cardiac function was determined by ultrasonography with ACUSON X700 ultrasound system (Siemens, Shinagawa-ku, Japan).

\section{Interventions}

All eligible patients were treated with routine regimens of perioperative intravenous hydration including continuous intravenous infusion of $\leq 1,000 \mathrm{~mL}$ of isotonic saline within $12 \mathrm{~h}$. Other perioperative interventions include: fluids mixed with medications and normal glucose or saline injections. In addition, each patient was encouraged to drink as much water as possible $24 \mathrm{~h}$ 
after PCI. For patients with systolic dysfunction or chronic heart function, hydration dosage was set at $1.5-2 \mathrm{~L} /$ day and $0.5 \mathrm{~mL} / \mathrm{kg} / \mathrm{h}$ in order to prevent decompensation of patients. The total oral hydration intake was recorded for all patients and the ratios of $\mathrm{OHV} / \mathrm{W}$ were calculated. During the period of oral hydration, the patients' responses were observed and inquired, and the clinical assessment of cardiac function and hemodynamic data were performed in real time. The use of diuretics by monitoring body fluids was based on a clinical assessment of cardiac function determined by cardiologists.

\section{Diagnostic Standards and Outcomes}

The occurrence of CI-AKI was defined as $\geq 0.5 \mathrm{mg} / \mathrm{dL}$ absolute or $\geq 25 \%$ relative increase in Scr within $48-72 \mathrm{~h}$ following PCI. The primary outcome was the effect of oral hydration vol/wt on CIAKI. The secondary outcomes were a composite of death and need for dialysis during hospitalization.

\section{Statistical Analysis}

Continuous variables were expressed as means \pm standard deviation or as the median (quartile) according to distribution. Categorical variables were expressed in frequency or as a percentage. These continuous variables were compared using Student's $t$ test or Wilcoxon rank-sum test according to distribution, and categorical variables were compared using the $\chi^{2}$ test, Fisher's exact test. Univariate and multivariate logistic regression models were used to evaluate the correlation between variables and CI-AKI. In addition, we further applied a multivariate piecewise linear regression model to examine the threshold effect of the OHV/W on CI-AKI by smooth curve fitting. Stratified logistic regression models were used for subgroup analysis. The interaction and modification between the subgroups were examined by likelihood ratio test. Data were analyzed with the use of statistical packages $\mathrm{R}$ (The R Foundation; http://www.r-project.org; version 3.4.3 2018-02-18) and EmpowerStats (www.empowerstats.com; X\&Y Solutions Inc., Boston, MA, USA).

\section{Results}

\section{The Demographic and Laboratory Data of the}

\section{Participants}

Overall, 754 participants were included in the analysis. The demographic and laboratory data were listed in Table 1.616 were male and 138 were female. The mean age of the subjects was $59.91 \pm 11.84$ years in patients without CI-AKI, and $62.65 \pm 14.09$ years in patients with CI-AKI. The total incidence of CI-AKI was $15.65 \%$ (118/754). The participants were divided into 2 groups: No CI-AKI group and CI-AKI group. Differences were found in terms of age, OHV/W, intravenous hydration volume (IVHV), number of stents and diabetes mellitus (DM). However, there were no differences in terms of gender, systolic pressure (SBP), diastolic pressure (DBP), BMI, left ventricular ejection fraction (LVEF), high blood pres-
Table 1. Baseline characteristics of participants

\begin{tabular}{|c|c|c|c|}
\hline Risk factors & No Cl-AKI & $\mathrm{Cl}-\mathrm{AKI}$ & $p$ value \\
\hline$N$ & 636 & 118 & - \\
\hline Age, years & $59.91 \pm 11.84$ & $62.65 \pm 14.09$ & 0.022 \\
\hline Gender male, $n$ (\%) & $526(82.70)$ & $90(76.27)$ & 0.097 \\
\hline $\mathrm{BMI}, \mathrm{kg} / \mathrm{m}^{2}$ & $24.62 \pm 3.71$ & $24.71 \pm 3.52$ & 0.817 \\
\hline BUN, mmol/L & $4.76 \pm 1.03$ & $4.84 \pm 1.00$ & 0.435 \\
\hline $\mathrm{UA}, \mu \mathrm{mol} / \mathrm{L}$ & $440.62 \pm 91.23$ & $452.44 \pm 86.29$ & 0.193 \\
\hline $\mathrm{TC}, \mathrm{mmol} / \mathrm{L}$ & $5.65 \pm 1.18$ & $5.78 \pm 1.00$ & 0.271 \\
\hline $\mathrm{HDL}, \mathrm{mmol} / \mathrm{L}$ & $1.31 \pm 0.25$ & $1.28 \pm 0.22$ & 0.223 \\
\hline LDL, mmol/L & $3.45 \pm 0.82$ & $3.47 \pm 0.89$ & 0.844 \\
\hline $\mathrm{TG}, \mathrm{mmol} / \mathrm{L}$ & $2.71 \pm 2.24$ & $3.09 \pm 3.35$ & 0.116 \\
\hline GLU, mmol/L & $5.77 \pm 1.76$ & $5.77 \pm 1.52$ & 0.984 \\
\hline SBP, mm Hg & $125.79 \pm 12.12$ & $126.15 \pm 11.62$ & 0.763 \\
\hline $\mathrm{DBP}, \mathrm{mm} \mathrm{Hg}$ & $78.11 \pm 8.29$ & $78.59 \pm 8.85$ & 0.565 \\
\hline LVEF, \% & $52.76 \pm 11.78$ & $50.63 \pm 11.33$ & 0.083 \\
\hline $\mathrm{Scr}, \mathrm{mmol} / \mathrm{L}$ & $80.03 \pm 15.68$ & $78.18 \pm 15.96$ & 0.240 \\
\hline $\mathrm{OHV} / \mathrm{W}, \mathrm{mL} / \mathrm{kg}$ & $19.89 \pm 9.37$ & $16.94 \pm 6.62$ & 0.001 \\
\hline IVHV, mL/24 h & $750(500-1,000)$ & $767(511-1,160)$ & 0.005 \\
\hline $\mathrm{DM}, n(\%)$ & $178(27.90)$ & $44(37.29)$ & 0.040 \\
\hline $\mathrm{HBP}, n(\%)$ & $258(40.44)$ & 46 (38.98) & 0.767 \\
\hline CCB, $n(\%)$ & $174(27.27)$ & $26(22.03)$ & 0.236 \\
\hline ACEI/ARB, $n(\%)$ & $268(42.01)$ & $56(47.46)$ & 0.272 \\
\hline$\beta$-Blocker, $n$ (\%) & 195 (30.66) & $36(30.51)$ & 0.974 \\
\hline Diuretics, $n(\%)$ & $54(8.491)$ & $8(6.780)$ & 0.715 \\
\hline Insulin, $n(\%)$ & $54(8.491)$ & $10(8.475)$ & 0.995 \\
\hline $\mathrm{OAD}, n(\%)$ & $134(21.069)$ & $34(28.814)$ & 0.063 \\
\hline \multicolumn{4}{|c|}{ Contrast agent, $n(\%)$} \\
\hline$\leq 100 \mathrm{~mL}$ & $456(71.7)$ & $86(72.88)$ & \multirow{2}{*}{0.793} \\
\hline$>100 \mathrm{~mL}$ & $180(28.3)$ & $32(27.12)$ & \\
\hline \multicolumn{4}{|l|}{ No. of stents, $n(\%)$} \\
\hline$\leq 1$ & $521(81.92)$ & $106(89.83)$ & \multirow{2}{*}{0.035} \\
\hline$>1$ & $115(18.08)$ & $12(10.17)$ & \\
\hline Diuretics, $n(\%)$ & $54(8.49)$ & $8(6.78)$ & 0.715 \\
\hline
\end{tabular}

BMI, body mass index; BUN, blood urea nitrogen; UA, uric acid; $\mathrm{TC}$, total cholesterol; HDL, high-density lipoprotein cholesterol; LDL, low-density lipoprotein cholesterol; TG, total cholesterol; GLU, blood glucose; SBP, systolic pressure; DBP, diastole pressure; LVEF, left ventricular ejection fraction; Scr, serum creatinine; OHV/W, oral hydration vol/wt; IVHV, intravenous hydration volume; DM, diabetes mellitus; HBP, high blood pressure; $\mathrm{CCB}$, calcium channel blocker; $A C E I / A R B$, angiotensin-converting enzyme inhibitor/angiotensin receptor blocker; OAD, oral antidiabetic drugs; $\mathrm{Cl}-\mathrm{AKI}$, contrastinduced acute kidney injury.

sure, lipidemia, blood glucose (GLU), baseline Scr, contrast agent, medications such as $\beta$-blocker, calcium channel blocker (CCB), angiotensin receptor blocker/angiotensin-converting enzyme inhibitor (ARB/ACEI), oral antidiabetic drugs (OAD), insulin, and diuretics. During hospitalization, no patient died or received renal replacement therapy. 
Table 2. Correlations between exposure factors and $\mathrm{Cl}-\mathrm{AKI}$

\begin{tabular}{|c|c|c|c|}
\hline Exposure & Statistic & OR (95\% Cl) & $p$ value \\
\hline Age, years & $60.34 \pm 12.25$ & $1.02(1.00,1.04)$ & 0.026 \\
\hline $\mathrm{BMI}, \mathrm{kg} / \mathrm{m}^{2}$ & $24.63 \pm 3.68$ & $1.01(0.95,1.06)$ & 0.817 \\
\hline BUN, mmol/L & $4.78 \pm 1.03$ & $1.08(0.83,1.41)$ & 0.563 \\
\hline $\mathrm{UA}, \mu \mathrm{mol} / \mathrm{L}$ & $442.64 \pm 90.53$ & $1.00(1.00,1.00)$ & 0.365 \\
\hline $\mathrm{TC}, \mathrm{mmol} / \mathrm{L}$ & $5.67 \pm 1.16$ & $1.09(0.87,1.37)$ & 0.435 \\
\hline $\mathrm{HDL}, \mathrm{mmol} / \mathrm{L}$ & $1.28 \pm 0.26$ & $2.16(0.76,6.15)$ & 0.151 \\
\hline $\mathrm{LDL}, \mathrm{mmol} / \mathrm{L}$ & $3.45 \pm 0.83$ & $1.03(0.74,1.43)$ & 0.873 \\
\hline $\mathrm{TG}, \mathrm{mmol} / \mathrm{L}$ & $2.77 \pm 2.45$ & $1.06(0.96,1.16)$ & 0.281 \\
\hline GLU, mmol/L & $5.77 \pm 1.73$ & $1.00(0.85,1.18)$ & 0.979 \\
\hline $\mathrm{SBP}, \mathrm{mm} \mathrm{Hg}$ & $125.84 \pm 12.03$ & $1.00(0.98,1.03)$ & 0.829 \\
\hline $\mathrm{DBP}, \mathrm{mm} \mathrm{Hg}$ & $78.17 \pm 8.39$ & $1.01(0.97,1.04)$ & 0.671 \\
\hline IVHV, mL/24 h & $750(500 \sim 1,000)$ & $1.000(1.000,1.001)$ & 0.153 \\
\hline LVEF, \% & $52.41 \pm 11.78$ & $0.98(0.96,1.01)$ & 0.228 \\
\hline $\mathrm{Scr}, \mathrm{mmol} / \mathrm{L}$ & $9.02 \pm 1.78$ & $0.99(0.98,1.01)$ & 0.392 \\
\hline \multicolumn{4}{|l|}{ Gender, $n(\%)$} \\
\hline Male & $616(81.70)$ & 1.0 & \multirow{2}{*}{0.099} \\
\hline Female & $138(18.30)$ & $1.49(0.93,2.38)$ & \\
\hline \multicolumn{4}{|c|}{ No. of stents, $n(\%)$} \\
\hline$\leq 1$ & $627(83.16)$ & 1.0 & \multirow{2}{*}{0.038} \\
\hline$>1$ & $127(16.84)$ & $0.51(0.27,0.96)$ & \\
\hline \multicolumn{4}{|l|}{$\mathrm{DM}, n(\%)$} \\
\hline No & $532(70.56)$ & 1.0 & \multirow[t]{2}{*}{0.043} \\
\hline Yes & $222(29.44)$ & $1.53(1.01,2.31)$ & \\
\hline \multicolumn{4}{|l|}{$\mathrm{HBP}, n(\%)$} \\
\hline No & 450 (59.68) & 1.0 & \multirow{2}{*}{0.748} \\
\hline Yes & $304(40.32)$ & $0.94(0.63,1.40)$ & \\
\hline \multicolumn{4}{|l|}{ CCB, $n(\%)$} \\
\hline No & $554(73.47)$ & 1.0 & \multirow{2}{*}{0.230} \\
\hline Yes & $200(26.53)$ & $0.75(0.47,1.20)$ & \\
\hline \multicolumn{4}{|l|}{ ACEI/ARB, $n(\%)$} \\
\hline No & $430(57.03)$ & 1.0 & \multirow{2}{*}{0.284} \\
\hline Yes & $324(42.97)$ & $1.24(0.84,1.84)$ & \\
\hline \multicolumn{4}{|l|}{$\beta$-Blocker, $n$ (\%) } \\
\hline No & $523(69.36)$ & 1.0 & \multirow{2}{*}{0.974} \\
\hline Yes & $231(30.64)$ & $0.99(0.65,1.52)$ & \\
\hline $\mathrm{OHV} / \mathrm{W}, \mathrm{mL} / \mathrm{kg}$ & $19.43 \pm 9.07$ & $0.96(0.93,0.99)$ & 0.023 \\
\hline \multicolumn{4}{|l|}{ Diuretics, $n(\%)$} \\
\hline No & $692(91.78)$ & 1.0 & \multirow{2}{*}{0.535} \\
\hline Yes & $62(8.22)$ & $0.78(0.36,1.69)$ & \\
\hline \multicolumn{4}{|l|}{ Insulin, $n$ (\%) } \\
\hline No & $690(91.51)$ & 1.0 & \multirow{2}{*}{0.995} \\
\hline Yes & $64(8.49)$ & $1.00(0.49,2.02)$ & \\
\hline \multicolumn{4}{|l|}{ OAD, $n(\%)$} \\
\hline No & $586(77.72)$ & 1.0 & \multirow{2}{*}{0.065} \\
\hline Yes & $168(22.28)$ & $1.52(0.97,2.36)$ & \\
\hline \multicolumn{4}{|c|}{ Contrast agent, $n(\%)$} \\
\hline$\leq 100 \mathrm{~mL}$ & $542(71.9)$ & 1.0 & \multirow{2}{*}{0.943} \\
\hline$>100 \mathrm{~mL}$ & $212(28.1)$ & $0.943(0.61,1.47)$ & \\
\hline
\end{tabular}

BMI, body mass index; BUN, blood urea nitrogen; UA, uric acid; TC, total cholesterol; HDL, high-density lipoprotein cholesterol; LDL, low-density lipoprotein cholesterol; TG, total cholesterol; GLU, blood glucose; SBP, systolic pressure; DBP, diastole pressure; LVEF, left ventricular ejection fraction; Scr, serum creatinine; OHV/W, oral hydration vol/wt; IVHV, intravenous hydration volume; DM, diabetic mellitus; $\mathrm{HBP}$, high blood pressure; $C C B$, calcium channel blocker; ACEI/ARB, angiotensin-converting enzyme inhibitor/angiotensin receptor blocker; $\mathrm{OAD}$, oral antidiabetic drugs; $\mathrm{Cl}-\mathrm{AKI}$, contrast-induced acute kidney injury. 
Table 3. Relationship between $\mathrm{OHV} / \mathrm{W}$ and $\mathrm{Cl}-\mathrm{AKI}$ in different models

\begin{tabular}{|c|c|c|c|c|c|c|}
\hline Variable & Model 1 OR $(95 \% \mathrm{CI})$ & $p$ value & Model 2 OR (95\% CI) & $p$ value & Model 3 OR (95\% CI) & $p$ value \\
\hline $\mathrm{OHV} / \mathrm{W}, \mathrm{mL} / \mathrm{kg}$ & $0.96(0.93,0.99)$ & 0.0225 & $0.96(0.93,1.00)$ & 0.0297 & $0.96(0.92,1.00)$ & 0.0503 \\
\hline \multicolumn{7}{|l|}{ OHV/W (trisection), mL/kg } \\
\hline$<12$ & 1.0 & & 1.0 & & 1.0 & 1.0 \\
\hline $12 \sim 24$ & $1.01(0.51,1.99)$ & 0.9861 & $1.04(0.52,2.08)$ & 0.9037 & $0.91(0.34,2.43)$ & 0.8492 \\
\hline$>24$ & $0.53(0.22,1.27)$ & 0.1531 & $0.55(0.23,1.31)$ & 0.1774 & $0.49(0.16,1.51)$ & 0.2158 \\
\hline
\end{tabular}

Model 1 was adjusted for: none. Model 2 was adjusted for: gender and age. Model 3 was adjusted for: gender, age, BMI, BUN, UA, TC, HDL, LDL, TG, GLU, SBP, DBP, LVEF, Scr, IVHV, DM, HBP, CCB, ACEI/ARB, No. of stents, diuretics; OAD and insulin. BMI, body mass index; BUN, blood urea nitrogen; $\mathrm{UA}$, uric acid; TC, total cholesterol; $\mathrm{HDL}$, high-density lipoprotein cholesterol; $\mathrm{LDL}$, low-density lipoprotein cholesterol; TG, total cholesterol; GLU, blood glucose; SBP, systolic pressure; DBP, diastole pressure; LVEF, left ventricular ejection fraction; Scr, serum creatinine; OHV/W, oral hydration vol/wt; IVHV, intravenous hydration volume; DM, diabetes mellitus; HBP, high blood pressure; CCB, calcium channel blocker; ACEI/ARB, angiotensin-converting enzyme inhibitor/angiotensin receptor blocker; OAD, oral antidiabetic drugs; $\mathrm{Cl}-\mathrm{AKI}$, contrast-induced acute kidney injury.

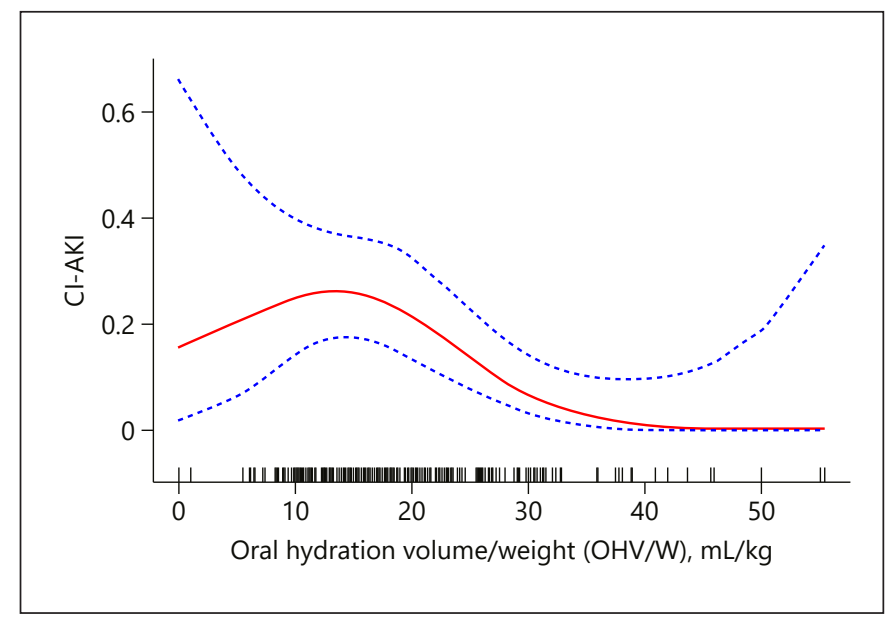

Fig. 2. The relationship between OHV/W and CI-AKI by smooth curve fitting. A nonlinear relationship between them was detected after adjusting for gender, age, BMI, BUN, UA, TC, HDL, LDL, TG, GLU, SBP, DBP, LVEF, Scr, IVHV, DM, HBP, CCB, ACEI/ $\mathrm{ARB}, \mathrm{No}$. of stents, diuretics, OAD and insulin. CI-AKI, contrastinduced acute kidney injury; OHV/W, oral hydration volume to weight ratio; BMI, body mass index; BUN, blood urea nitrogen; UA, uric acid; TC, total cholesterol; HDL, high-density lipoprotein cholesterol; LDL, low-density lipoprotein cholesterol; TG, total cholesterol; GLU, blood glucose; SBP, systolic pressure; DBP, diastole pressure; LVEF, left ventricular ejection fraction; Scr, serum creatinine; OHV/W, oral hydration vol/wt; IVHV, intravenous hydration volume; DM, diabetes mellitus; HBP, high blood pressure; $\mathrm{CCB}$, calcium channel blocker; $\mathrm{ACEI} / \mathrm{ARB}$, angiotensin-converting enzyme inhibitor/angiotensin receptor blocker; $\mathrm{OAD}$, oral antidiabetic drugs.

\section{Univariate Analysis}

We used univariate analysis model to evaluate the associations between exposure factors and CI-AKI, and the results showed that the factors related to CI-AKI were OHV/W (OR $=0.96,95 \% \mathrm{CI}: 0.93 \sim 0.99, p=0.023)$, age $(\mathrm{OR}=1.02,95 \% \mathrm{CI}: 1.00 \sim 1.04, p=0.026)$, No. of stents $(\mathrm{OR}=0.51,95 \%$ CI: 0.27 0.96, $p=0.038), \mathrm{DM}(\mathrm{OR}=$ 1.53, 95\% CI: $1.01 \sim 2.31, p=0.043)$. However, BMI, SBP, DBP, LVEF, IVHV, lipidemia, GLU, Scr, number of stents, contrast agent, and medications such as $\beta$-blocker, ACEI/ARB, CCB, OAD, insulin, and diuretics were not associated with CI-AKI (All $p>0.05)$ (Table 2).

\section{The Effect Analysis of OHV/W on CI-AKI Using Multiple Logistic Regression}

Multiple logistic regression model was performed to investigate the correlation between OHV/W and CI-AKI. Unadjusted and adjusted models were shown in the Table 3. In model 1 (unadjusted model), result showed a negative association with CI-AKI (OR $=0.96,95 \% \mathrm{CI}$ : $0.93 \sim 0.99, p=0.0225$ ). In addition, the negative association was also observed in model 2 (adjusted for age and gender) between OHV/W and CI-AKI (OR $=0.96,95 \%$ CI: $0.93 \sim 1.00, p=0.0297$ ). However, in the model 3 (fully adjusted model), there was no correlation between OHV/W and CI-AKI (OR = 0.96, 95\% CI: 0.92 1.00, $p=$ $0.0503)$. For further sensitivity analysis, we also treated $\mathrm{OHV} / \mathrm{W}$ as a categorical variable, and found nonassociation between OHV/W and CI-AKI in model 1 to model 3 (all $p>0.05)$. 
Table 4. The independent correlation between OHV/W and $\mathrm{Cl}-\mathrm{AKI}$ by multivariate piecewise linear regression

\begin{tabular}{|c|c|c|c|c|}
\hline \multirow{2}{*}{$\begin{array}{l}\text { Infection point of } \\
\mathrm{OHV} / \mathrm{W}, \mathrm{mL} / \mathrm{kg}\end{array}$} & \multirow{2}{*}{$\begin{array}{l}\text { Effect } \\
\text { size (HR) }\end{array}$} & \multicolumn{2}{|c|}{$95 \% \mathrm{Cl}$} & \multirow[t]{2}{*}{$p$ value } \\
\hline & & lower & upper & \\
\hline$<15.69$ & 1.19 & 0.95 & 1.49 & 0.1302 \\
\hline$\geq 15.69$ & 0.90 & 0.82 & 0.98 & 0.0126 \\
\hline
\end{tabular}

Adjusting for gender, age, BMI, BUN, UA, TC, HDL, LDL, TG, GLU, SBP, DBP, LVEF, SCr, IVHV, DM, HBP, CCB, ACEI/ARB, No. of stents, diuretics; $O A D$ and insulin. $B M I$, body mass index; $B U N$, blood urea nitrogen; UA, uric acid; TC, total cholesterol; $\mathrm{HDL}$, high-density lipoprotein cholesterol; LDL, low-density lipoprotein cholesterol; TG, total cholesterol; GLU, blood glucose; SBP, systolic pressure; DBP, diastole pressure; LVEF, left ventricular ejection fraction; Scr, serum creatinine; OHV/W, oral hydration vol/wt; IVHV, intravenous hydration volume; $\mathrm{DM}$, diabetes mellitus; $\mathrm{HBP}$, high blood pressure; $C C B$, calcium channel blocker; ACEl/ARB, angiotensin-converting enzyme inhibitor/angiotensin receptor blocker; OAD, oral antidiabetic drugs; $\mathrm{Cl}-\mathrm{AKI}$, contrast-induced acute kidney injury.

\section{The Effect Analysis of OHV/W on CI-AKI Using}

Piecewise Linear Regression

Since OHV/W was continuous variable, it was necessary to use nonlinear regression analysis. In the present study (Fig. 2), nonlinear relationship was detected between OHV/W and CI-AKI (adjusted for age, BMI, gender, blood urea nitrogen, uric acid, total cholesterol, highdensity lipoprotein cholesterol, low-density lipoprotein cholesterol, triglyceride, GLU, SBP, DBP, LVEF, Scr, IVHV, DM, high blood pressure, CCB, ACEI/ARB, diuretics, $\mathrm{OAD}$ and insulin). By 2-piecewise linear regression model, the inflection point was $15.69 \mathrm{~mL} / \mathrm{kg}$. On the right side of the inflection point $(\mathrm{OHV} / \mathrm{W} \geq 15.69 \mathrm{~mL} /$ $\mathrm{kg}$ ), a negative relationship was detected between $\mathrm{OHV} / \mathrm{W}$ and CI-AKI (HR $=0.90,95 \%$ CI: 0.82 0.98, $p=0.0126$ ). However, the relationship between OHV/W and CI-AKI on the left of inflection point was not observed (HR = 1.19, 95\% CI: $0.95 \sim 1.49, p=0.1302$ ) (Table 4 ).

\section{The Effect of $\mathrm{OHV} / \mathrm{W}$ on CI-AKI with Subgroup Analyses}

To further explore the relationship between OHV/W and CI-AKI, we analyzed the subgroup (Table 5). Stratified analyses were performed by age $(<60, \geq 60$ years), gender, BMI $\left(<25, \geq 25 \mathrm{~kg} / \mathrm{m}^{2}\right)$, drugs including CCB, $\mathrm{ARB} / \mathrm{ACEI}$, diuretics, $\beta$-blocker, $\mathrm{OAD}$, and insulin (yes/ no). Subgroup analysis showed that significant interactions were observed only for gender difference ( $p$ for interaction $=0.0155$ ), male patients had a significantly low- er risk of CI-AKI ( $\mathrm{HR}=0.84,95 \%$ CI: $0.75 \sim 0.93, p=$ $0.0012)$. However, the test for interactions were not statistically significant for age, BMI and drugs including $\mathrm{CCB}, \mathrm{ARB} / \mathrm{ACEI}$, diuretics, $\beta$-blocker and OAD ( $p$ for all interactions $>0.05$ ).

\section{Discussion}

The present study was to examine the protection effect of OHV/W on CI-AKI among patients with STEMI treated with PCI. As shown in multiple logistic regression model (fully adjusted), OHV/W was not correlation with CI-AKI. However, we further found that there was a nonlinear relationship between $\mathrm{OHV} / \mathrm{W}$ and CIAKI. The different correlations of OHV/W and CI-AKI were found on the left and right sides of inflection point $(\mathrm{OHV} / \mathrm{W}=15.69 \mathrm{~mL} / \mathrm{kg})$. On the right side of the inflection point $(\mathrm{OHV} / \mathrm{W} \geq 15.69 \mathrm{~mL} / \mathrm{kg})$, a negative relationship was detected between $\mathrm{OHV} / \mathrm{W}$ and CI-AKI $(\mathrm{HR}=0.90,95 \%$ CI: $0.82-0.98, p=0.0126)$. However, the relationship between OHV/W and CI-AKI on the left of inflection point was not observed $(\mathrm{HR}=1.19$, 95\% CI: $0.95 \sim 1.49, p=0.1302$ ). Subgroup analysis showed that male patients had a significantly lower risk of CI-AKI.

CI-AKI was a complication of angiographical procedures [16]. Severe CI-AKI not only prolonged hospitalization, increased the cost of medical treatment, but also increased the mortality of patients [3, 17, 18]. 2018 ESC/EACTS Guidelines recommend that adequate hydration remains the chief measure to prevent CI-AKI [19]. However, there was limited research on CI-AKI caused by PCI in emergency [20]. Taylor et al. [21] reported that for patients with mild to moderate renal dysfunction undergoing elective cardiac catheterization, the efficacy of oral hydration was similar to that of intravenous hydration in the prevention of CI-AKI. In 2006, Dussol et al. [22] conducted a small sample, randomized controlled trial, which proved that oral saline hydration and intravenous saline hydration were equally effective in preventing CI-AKI in patients with chronic kidney disease. Recently, Cho et al. [23] compared 2 methods of oral hydration and intravenous hydration, and the results showed that the incidence of CI-AKI, hospital stay, mortality, and other complications were similar. Wrobel et al. [24] compared 102 patients with diabetes undergoing coronary angiography and showed that oral mineral water was as efficient as intravenous hydration. Likewise, another study showed 
Table 5. Subgroup analysis of the associations between $\mathrm{OHV} / \mathrm{W}$ and $\mathrm{Cl}-\mathrm{AKI}$

\begin{tabular}{|c|c|c|c|c|c|}
\hline \multirow[t]{2}{*}{ Characteristic } & \multirow{2}{*}{$\begin{array}{l}\text { Effect } \\
\text { size (HR) }\end{array}$} & \multicolumn{2}{|c|}{$95 \% \mathrm{Cl}$} & \multirow[t]{2}{*}{$p$ value } & \multirow{2}{*}{$\begin{array}{l}p \\
\text { for interaction }\end{array}$} \\
\hline & & lower & upper & & \\
\hline \multicolumn{6}{|l|}{ Age } \\
\hline$<60$ years & 0.96 & 0.91 & 1.01 & 0.0871 & \multirow{2}{*}{0.5252} \\
\hline$\geq 60$ years & 0.94 & 0.90 & 0.98 & 0.0028 & \\
\hline \multicolumn{6}{|l|}{ Gender } \\
\hline Male & 0.84 & 0.75 & 0.93 & 0.0012 & \multirow{2}{*}{0.0155} \\
\hline Female & 0.95 & 0.91 & 0.99 & 0.0125 & \\
\hline \multicolumn{6}{|l|}{ BMI } \\
\hline$<25$ & 0.92 & 0.87 & 0.96 & 0.0005 & \multirow{2}{*}{0.0541} \\
\hline$\geq 25$ & 0.99 & 0.93 & 1.05 & 0.6834 & \\
\hline \multicolumn{6}{|l|}{ CCB } \\
\hline No & 0.96 & 0.92 & 0.99 & 0.0198 & \multirow{2}{*}{0.3275} \\
\hline Yes & 0.91 & 0.83 & 1.00 & 0.0548 & \\
\hline \multicolumn{6}{|l|}{ ARB/ACEI } \\
\hline No & 0.90 & 0.85 & 0.96 & 0.022 & \multirow{2}{*}{0.2610} \\
\hline Yes & 0.95 & 0.90 & 1.00 & 0.035 & \\
\hline \multicolumn{6}{|l|}{ Diuretics } \\
\hline No & 0.96 & 0.922 & 1.003 & 0.0667 & \multirow{2}{*}{0.6919} \\
\hline Yes & 0.94 & 0.819 & 1.069 & 0.3299 & \\
\hline \multicolumn{6}{|l|}{$\beta$-Blocker } \\
\hline No & 0.97 & 0.94 & 1.00 & 0.0785 & \multirow{2}{*}{0.0718} \\
\hline Yes & 0.92 & 0.86 & 0.97 & 0.0051 & \\
\hline \multicolumn{6}{|l|}{ OAD } \\
\hline No & 0.97 & 0.93 & 1.01 & 0.0938 & \multirow{2}{*}{0.0728} \\
\hline Yes & 0.89 & 0.82 & 0.97 & 0.0100 & \\
\hline \multicolumn{6}{|l|}{ Insulin } \\
\hline No & 0.96 & 0.92 & 0.99 & 0.084 & \multirow{2}{*}{0.0520} \\
\hline Yes & 0.82 & 0.69 & 0.97 & 0.0207 & \\
\hline
\end{tabular}

Above model adjusted for gender, age, BMI, BUN, UA, TC, HDL, LDL, TG, GLU, SBP, DBP, LVEF, Scr, IVHV, DM, HBP, CCB, ACEI/ARB, No. of stents, diuretics; OAD and insulin. In each case, the model is not adjusted for the stratification variable. BMI, body mass index; BUN, blood urea nitrogen; UA, uric acid; TC, total cholesterol; HDL, high-density lipoprotein cholesterol; LDL, low-density lipoprotein cholesterol; TG, total cholesterol; GLU, blood glucose; SBP, systolic pressure; DBP, diastole pressure; LVEF, left ventricular ejection fraction; $\mathrm{Scr}$, serum creatinine; OHV/W, oral hydration vol/wt; IVHV, intravenous hydration volume; DM, diabetes mellitus; HBP, high blood pressure; CCB, calcium channel blocker; ACEl/ARB, angiotensin-converting enzyme inhibitor/angiotensin receptor blocker; OAD, oral antidiabetic drugs; $\mathrm{Cl}-\mathrm{AKI}$, contrast-induced acute kidney injury. that oral hydration may be as effective as intravenous hydration in the prevention of contrast-induced nephropathy [25]. However, these studies [20-25] were only aimed at patients with nonemergency coronary angiography or PCI, and the hydration scheme was variable, which had insufficient significance for clinical practice. Moreover, little was known about oral hydration strategy on prevention of a high-risk population such as STEMI patients following PCI.

The ratio of $\mathrm{CM}$ /estimated glomerular filtration rate is one of the most commonly used indexes to predict CIAKI in patients with STEMI undergoing PCI. Studies have shown that the cutoff value of the CM/estimated glomerular filtration rate was 3.7 , which is a strong independent predictor of CI-AKI [26, 27]. However, no literature on the cutoff value of the protective effect of oral hydration on CI-AKI was conducted. Song et al. [28] examined the protective effect of the OHV/W on CI-AKI among patients with STEMI after PCI. They found that $\mathrm{OHV} / \mathrm{W}$ ratio $\geq 12 \mathrm{~mL} / \mathrm{kg}$ was an independent protective factor associated with CI-AKI (OR $=0.349,95 \% \mathrm{CI}$ : $0.147 \sim 0.828, p=0.017)$. The value of oral hydration in the study was set as $12 \mathrm{~mL} / \mathrm{kg}$ artificially, and logistic analysis was used to analyze the effect of oral hydration 
volume $(12 \mathrm{~mL} / \mathrm{kg})$ on CI-AKI. However, in our study, in addition to using logistic model, we also used generalized additive model (GAM) to evaluate the nonlinear relationship between $\mathrm{OHV} / \mathrm{W}$ and CI-AKI. The results showed that OHV/W was nonlinear correlated with CIAKI. GAM has obvious advantages over logistic model in dealing with nonlinear relations. It can automatically estimate the function (curve) relations of each prediction term by using data [29]. Obviously, the use of GAM will help us better discover the real relationship between $\mathrm{OHV} / \mathrm{W}$ and CI-AKI.

Although the previous study showed a linear association between $\mathrm{OHV} / \mathrm{W}$ and CI-AKI, our study did not find this relationship after adjusting for age, gender, intravenous hydration volume, number of stents, medications, and other potential confounders. In view of the nonlinear relationship between $\mathrm{OHV} / \mathrm{W}$ and CI-AKI, further subgroup analysis was needed. The results of subgroup analysis showed that there was a significant interactive effect of $\mathrm{OHV} / \mathrm{W}$ and gender difference ( $p$ for interaction $=0.0155)$, male patients had a significantly lower risk of CI-AKI (HR $=0.84,95 \%$ CI: $0.75 \sim 0.93, p=0.0012$ ). Our findings suggested that male patients may particularly benefit from $\mathrm{OHV}$ to prevent CI-AKI.

Our research showed that $\mathrm{OHV} / \mathrm{W} \geq 15.6 \mathrm{~mL} / \mathrm{kg}$ for 24 $\mathrm{h}$ post-procedure may be an effective preventive strategy of CI-AKI. However, overload of oral hydration capacity should be avoided, especially in patients with systolic dysfunction or chronic heart function. Our strategy was that hydration dosage was set at $0.5 \mathrm{~mL} / \mathrm{kg} / \mathrm{h}$ in order to prevent decompensation of patients. Our results showed that there was no heart failure or renal replacement therapy and no patient died during the hospitalization period, suggesting the safety of oral hydration.

There were some limitations of the study: (1) the present study was conducted retrospectively in a single-center, even though we adjusted potential confounding factors with the GAM model. Some selection bias must be noted; (2) data regarding concomitant use of statins were not present for participants, and their impact on the development of CI-AKI cannot be assessed; (3) variations in the times at which the measurements were undertaken may have missed the post-procedural peak creatinine levels; (4) all participants received routine intravenous hydration. Hemodilution can reduce Scr, and cumulative daily fluid balance directly affects the concentration of Scr, and may finally affect the conclusion.

\section{Conclusion}

Our study showed a nonlinear relationship between $\mathrm{OHV} / \mathrm{W}$ and CI-AKI in patients with STEMI after adjusting for potential confounding factors. This finding suggests that $\mathrm{OHV} / \mathrm{W} \geq 15.69 \mathrm{~mL} / \mathrm{kg}$ for $24 \mathrm{~h}$ post-procedure may be an effective preventive strategy of CI-AKI. In addition, male patients may particularly benefit from $\mathrm{OHV}$ to prevent CI-AKI.

\section{Acknowledgments}

We would like to thank the participants in this study for their cooperation. We also thank the academic and nonacademic staff of Guangdong Provincial Hospital of Integrated Traditional Chinese and Western Medicine for their help.

\section{Statement of Ethics}

The study was approved by the Ethics Committee of Guangdong Province Hospital of Integrated Traditional Chinese and Western Medicine. This was a retrospective study that did not need informed consent.

\section{Conflict of Interest Statement}

The authors declare no potential conflict of interests.

\section{Funding Sources}

There was no funding support for this research.

\section{Author Contributions}

Weining Xie contributed to acquisition, analysis, and interpretation of data and took responsibility for the construction of the whole of the manuscript. Yuge Zhou contributed to acquisition and analysis of data, drafting the manuscript, and literature review. Zhishan Liao contributed to acquisition, analysis, and interpretation of data. Biying Lin designed the work, constructed an idea for the research, supervised the course of the project, and reviewed the article.

\section{Data Availability Statement}

The data used to support the findings of the study can be made available upon request to the corresponding author. 


\section{References}

1 Thomsen HS, Morcos SK, Barrett BJ. Contrast-induced nephropathy: the wheel has turned 360 degrees. Acta Radiol. 2008;49(6): 646-57.

2 Nash K, Hafeez A, Hou S. Hospital-acquired renal insufficiency. Am J Kidney Dis. 2002; 39(5):930-6.

3 James MT, Ghali WA, Knudtson ML, Ravani P, Tonelli M, Faris P, et al. Associations between acute kidney injury and cardiovascular and renal outcomes after coronary angiography. Circulation. 2011;123(4):409-16.

4 Marenzi G, Lauri G, Assanelli E, Campodonico J, De Metrio M, Marana I, et al. Contrastinduced nephropathy in patients undergoing primary angioplasty for acute myocardial infarction. J Am Coll Cardiol. 2004;44(9):17805

5 Pyxaras SA, Sinagra G, Mangiacapra F, Perkan A, Di Serafino L, Vitrella G, et al. Contrast-induced nephropathy in patients undergoing primary percutaneous coronary intervention without acute left ventricular ejection fraction impairment. Am J Cardiol. 2013; 111(5):684-8

6 Seeliger E, Sendeski M, Rihal CS, Persson PB. Contrast-induced kidney injury: mechanisms, risk factors, and prevention. Eur Heart J. 2012;33(16):2007-15.

7 Rear R, Bell RM, Hausenloy DJ. Contrast-induced nephropathy following angiography and cardiac interventions. Heart. 2016; 102(8):638-48

8 Weisbord SD, Gallagher M, Kaufman J, Cass A, Parikh CR, Chertow GM, et al. Prevention of contrast-induced AKI: a review of published trials and the design of the prevention of serious adverse events following angiography (PRESERVE) trial. Clin J Am Soc Nephrol. 2013;8(9):1618-31.

9 Fähling M, Seeliger E, Patzak A, Persson PB. Understanding and preventing contrast-induced acute kidney injury. Nat Rev Nephrol. 2017;13(3):169-80.

10 Jiang Y, Chen M, Zhang Y, Zhang N, Yang H, Yao J, et al. Meta-analysis of prophylactic hydration versus no hydration on contrast-induced acute kidney injury. Coron Artery Dis. 2017;28(8):649-57.

11 Narula A, Mehran R, Weisz G, Dangas GD, $\mathrm{Yu}$ J, Généreux $\mathrm{P}$, et al. Contrast-induced acute kidney injury after primary percutaneous coronary intervention: results from the HORIZONS-AMI substudy. Eur Heart J. 2014;35(23):1533-40.
12 Marenzi G, Cabiati A, Bertoli SV, Assanelli E, Marana I, De Metrio M, et al. Incidence and relevance of acute kidney injury in patients hospitalized with acute coronary syndromes. Am J Cardiol. 2013;111(6):816-22.

13 Watabe H, Sato A, Hoshi T, Takeyasu N, Abe D, Akiyama D, et al. Association of contrastinduced acute kidney injury with long-term cardiovascular events in acute coronary syndrome patients with chronic kidney disease undergoing emergent percutaneous coronary intervention. Int J Cardiol. 2014;174(1):5763.

14 Queiroz RE, de Oliveira LS, de Albuquerque CA, Santana CA, Brasil PM, Carneiro LL, et al. Acute kidney injury risk in patients with ST-segment elevation myocardial infarction at presentation to the ED. Am J Emerg Med. 2012;30(9):1921-7.

15 O'Gara PT, Kushner FG, Ascheim DD, Casey DE Jr, Chung MK, de Lemos JA, et al. 2013 ACCF/AHA guideline for the management of ST-elevation myocardial infarction: a report of the American College of Cardiology Foundation/American Heart Association task force on practice guidelines. Circulation. 2013;127(4):e362-425.

16 Singri N, Ahya SN, Levin ML. Acute renal failure. JAMA. 2003;289(6):747-51.

17 McCullough PA, Wolyn R, Rocher LL, Levin $\mathrm{RN}$, O'Neill WW. Acute renal failure after coronary intervention: incidence, risk factors, and relationship to mortality. Am J Med. 1997;103(5):368-75

18 Tepel M, Aspelin P, Lameire N. Contrast-induced nephropathy: a clinical and evidencebased approach. Circulation. 2006;113(14): 1799-806.

19 Neumann FJ, Sousa-Uva M, Ahlsson A, Alfonso F, Banning AP, Benedetto U, et al. [2018 ESC/EACTS Guidelines on myocardial revascularization. The task force on myocardial revascularization of the European Society of Cardiology (ESC) and European Association for Cardio-Thoracic Surgery (EACTS)]. G Ital Cardiol. 2019;20(2):1S-61S.

20 Akyuz S, Karaca M, Kemaloglu Oz T, Altay S, Gungor B, Yaylak B, et al. Efficacy of oral hydration in the prevention of contrast-induced acute kidney injury in patients undergoing coronary angiography or intervention. Nephron Clin Pract. 2014;128(1-2):95-100.
21 Taylor AJ, Hotchkiss D, Morse RW, McCabe J. PREPARED: preparation for angiography in renal dysfunction: a randomized trial of inpatient vs outpatient hydration protocols for cardiac catheterization in mild-to-moderate renal dysfunction. Chest. 1998;114(6):1570 4.

22 Dussol B, Morange S, Loundoun A, Auquier $\mathrm{P}$, Berland Y. A randomized trial of saline hydration to prevent contrast nephropathy in chronic renal failure patients. Nephrol Dial Transplant. 2006;21(8):2120-6.

23 Cho R, Javed N, Traub D, Kodali S, Atem F, Srinivasan V. Oral hydration and alkalinization is noninferior to intravenous therapy for prevention of contrast-induced nephropathy in patients with chronic kidney disease. J Interv Cardiol. 2010;23(5):460-6.

24 Wróbel W, Sinkiewicz W, Gordon M Woźniak-Wiśniewska A. Oral versus intravenous hydration and renal function in diabetic patients undergoing percutaneous coronary interventions. Kardiol Pol. 2010;68(9):101520.

25 Trivedi HS, Moore H, Nasr S, Aggarwal K, Agrawal A, Goel P, et al. A randomized prospective trial to assess the role of saline hydration on the development of contrast nephrotoxicity. Nephron Clin Pract. 2003;93(1): C29-34.

26 Laskey WK, Jenkins C, Selzer F, Marroquin OC, Wilensky RL, Glaser R, et al. Volume-tocreatinine clearance ratio: a pharmacokinetically based risk factor for prediction of early creatinine increase after percutaneous coronary intervention. J Am Coll Cardiol. 2007; 50(7):584-90.

27 Mager A, Vaknin Assa H, Lev EI, Bental T, Assali A, Kornowski R. The ratio of contrast volume to glomerular filtration rate predicts outcomes after percutaneous coronary intervention for ST-segment elevation acute myocardial infarction. Catheter Cardiovasc Interv. 2011;78(2):198-201.

28 Song F, Sun G, Liu J, Chen JY, He Y, Chen S, et al. The association between post-procedural oral hydration and risk of contrast-induced acute kidney injury among ST-elevation myocardial infarction patients undergoing primary percutaneous coronary intervention. Ann Transl Med. 2019;7(14):321.

29 Vislocky RL, Fritsch JM. Generalized additive models versus linear regression in generating probabilistic MOS forecasts of aviation weather parameters. Weather Forecast. 1995; 10(4):669-80. 